\title{
X-ray Spectroscopies of High Energy Density Matter Created with X-ray Free Electron Lasers
}

\author{
Byoung Ick Cho ${ }^{1,2}$ \\ 1 Center for Relativistic Laser Science, Institute for Basic Science (IBS), Gwangju 61005, Korea; \\ bicho@gist.ac.kr; Tel.: +82-62-715-2879 \\ 2 Department of Physics and Photon Science, Gwangju Institute of Science and Technology (GIST), \\ Gwangju 61005, Korea
}

Received: 9 October 2019; Accepted: 7 November 2019; Published: 10 November 2019

\begin{abstract}
The recent progress in the development of X-ray free electron lasers (XFELs) allows for the delivery of over $10^{11}$ high-energy photons to solid-density samples in a femtosecond time scale. The corresponding peak brightness of XFEL induces a nonlinear response of matter in a short-wavelength regime. The absorption of an XFEL pulse in a solid also results in the creation of high energy density (HED) matter. The electronic structure and related fundamental properties of such HED matter can be investigated with the control of XFEL and various X-ray spectroscopic techniques. These experimental data provide unique opportunities to benchmark theories and models for extreme conditions and to guide further advances. In this article, the current progress in spectroscopic studies on intense XFEL-matter interactions and HED matter are reviewed, and future research opportunities are discussed.
\end{abstract}

Keywords: X-ray free electron laser; high energy density matter; X-ray spectroscopy

\section{Introduction}

The recent progress in development of short-wavelength free-electron lasers (FELs) has opened up a variety of opportunities in diverse fields such as atomic physics, plasma physics, ultrafast chemistry, and biomolecular imaging [1-4]. The parameter that distinguishes FELs the most from other light sources is the peak brightness, which is about a billion times greater than that of synchrotrons. Handling light intensity in excess of $10^{16} \mathrm{~W} / \mathrm{cm}^{2}$, which had been available only in infrared and optical regimes, has become feasible in EUV and X-ray regimes, and a nonlinear response of matter involving core electrons in femtosecond scales can be expected. Materials absorbing large numbers of X-ray photons are also anticipated to occur in extreme temperature and pressure conditions. Under such extreme environments, normal matter behaves abnormally and often regulates the physical processes in stars and large planets [5], inertial confinement fusion [6], as well as the applied processes of laser machining and ablation [7].

High energy density (HED) science is defined as the study of matter at extreme radiation, pressure, and temperature corresponding to energy densities in excess of about $10^{11} \mathrm{~J} / \mathrm{m}^{3}[8,9]$. As illustrated in Figure 1, HED conditions are widespread in the universe and laboratories. Within the regime of HED states, warm dense matter (WDM) representing the state where the thermal energy is comparable to the Fermi energy, the ions are strongly coupled. 


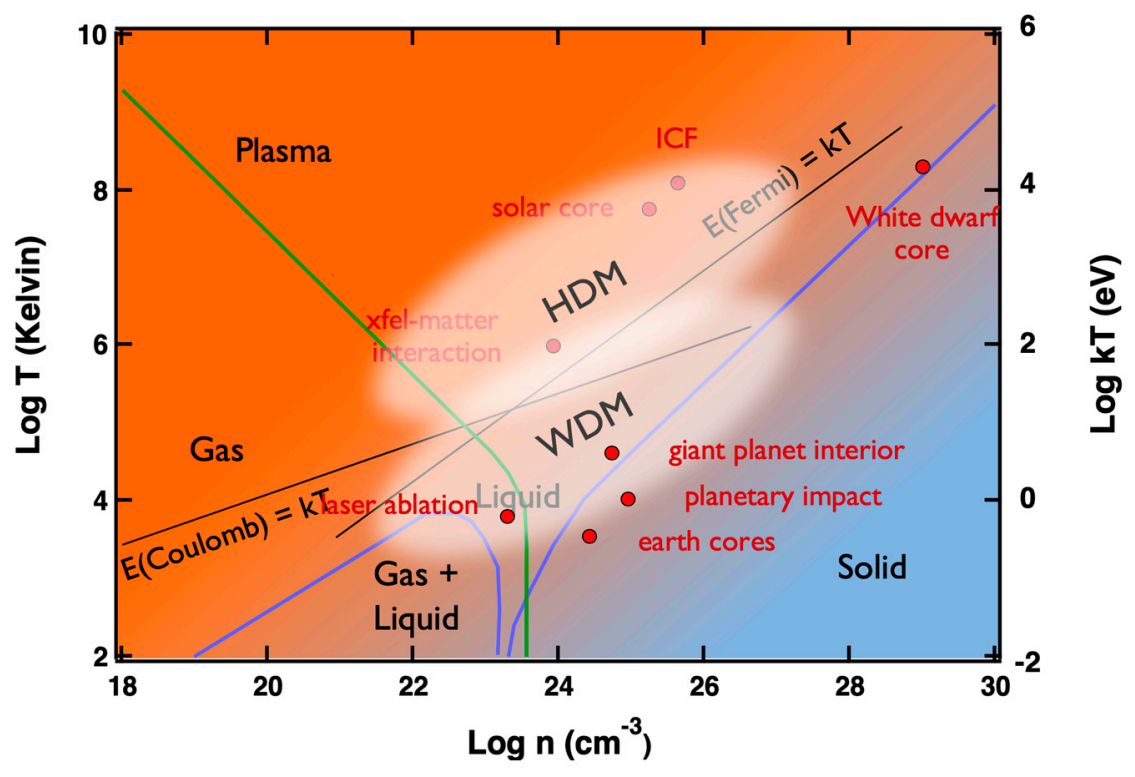

Figure 1. Phase diagram of warm and hot dense matter. WDM and HDM lie at the confluence of plasma and condensed matter. The orange shaded region indicates the domain of non-degenerate matter mainly described by the classical physics, while the blue region indicates the domain of strongly degenerate matter, where the quantum phenomena are dominant. In WDM and HDM, both classical and quantum effects are important, but not described adequately. Temperatures and densities of various structures in the universe and laboratories fall into these regimes. The figure is reproduced from [9].

$$
\Gamma=\frac{Z^{2} e^{2}}{r_{0} k_{B} T} \geq 1
$$

Here, $\Gamma$ is the plasma coupling parameter. When $\Gamma$ is an order of unity or greater, the plasma is considered as strongly coupled. $Z$ is the ion charge, and $r_{0}$ is the inter-particle distance. In general, WDM is partially ionized, and free electrons are also partially degenerated. Hot dense matter (HDM) represents the states with higher temperature with similar density. Owing to the sufficiently high temperatures, in HDM, a variety of charge states are assembled and free electrons are no longer degenerated $[10,11]$. As intermediate states of matter between the well-defined disciplines of condensed matter and plasmas, the investigation of WDM and HDM provides key understanding of non-equilibrium phase transitions and energy relaxation processes in extreme conditions. However, the complicated interplay of the physical processes in HED matter is difficult to describe.

Quantitative research on HED materials requires the ability to generate and diagnose well-defined high-temperature, high-density, and high-pressure conditions. Generally, they can be obtained by focusing a high-power laser or particle beam onto dense targets. However, such extreme matter is often non-uniform and quickly transforms to low-density plasma in a timescale of femtosecond to nanoseconds. The material properties are often measured for multi-stages instead of a single state. Optical diagnostics using femtosecond lasers have been the fastest means but could provide limited information about the surface of dense matter and outer electrons. The lack of experimental techniques to quantify single-state parameters at various temperature-density conditions has posed obstacles in the measurement of the fundamental properties and testing the theoretical description of simple materials such as hydrogen, carbon, and gold in the HED states.

In this context, the development of XFEL was an important step for advancing HED science. XFEL can heat solid samples to a temperature of millions of degrees to produce WDM and HDM, unlike any previous device. The typical long penetration length of $X$-rays enables it to heat a larger volume (thickness) of the sample than the optical lasers. Owing to the femtosecond pulse duration, this heating 
is isochoric with an initial density. These set well-defined density and temperature conditions in a larger volume of the sample with improved uniformity. XFEL also provides unique opportunities to study extreme materials created using other sources with various ultrafast techniques such as diffraction, scattering, imaging, and spectroscopy at short-wavelengths [12-17]. The unprecedented peak brightness of XFEL also induces the nonlinear responses of matter, which were hardly observed in the short-wavelength regime. With further development of seeded and attosecond FEL pulses, a coherent $x$-ray source can be applied to exploit quantum effects in the dense plasma environment $[18,19]$.

In the following sections, some of the first spectroscopic studies on intense FEL-matter interactions and HED matter created as a result (in the last decade) are reviewed. X-ray emission and absorption spectroscopies (XES/XAS) are simple but powerful diagnostics and are complimentary to each other in that they probe occupied and unoccupied electronic structures, as well as study the response to the radiations. Findings from these investigations and the advances in our understanding of the HED regime will be discussed.

\section{General Concept of Investigation}

Figure 2a shows the general sketch of the experiment. For efficient heating, a large number of X-ray photons need to be focused tightly. Using advanced focusing optics, such as Kirkpatrick-Baez mirror and compound refractive lens (CRL), XFEL beam can be focused to micro- to nano-meter sizes, and X-ray peak intensity in excess of $10^{16} \mathrm{~W} / \mathrm{cm}^{2}$ can be achieved [20]. It is noted that the laser intensity of $3 \times 10^{16} \mathrm{~W} / \mathrm{cm}^{2}$ correspondsto the Coulomb field which binds 1s electron in a hydrogen atom $\left(5 \times 10^{11} \mathrm{~V} / \mathrm{m}\right)$, and prior to XFELs, such light intensity was only available in the infrared and optical regimes. As samples, free-standing foils are the simplest cases. However, other forms, such as multi-layered targets [21] and liquid droplets [22], can also be used.

(a)

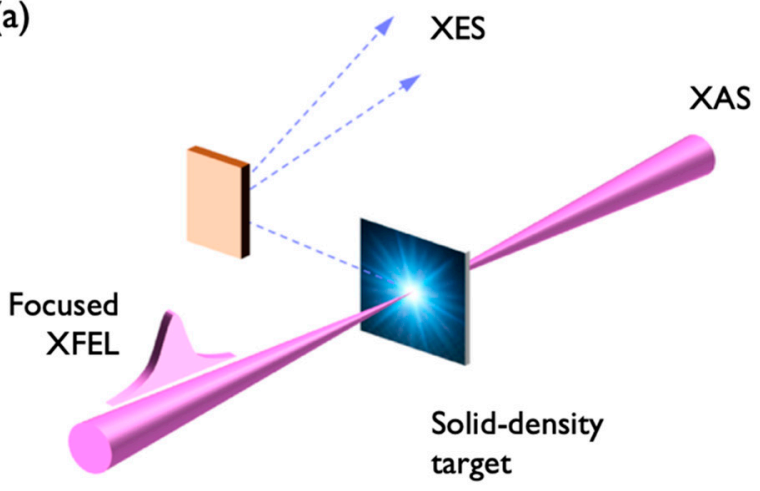

(b)

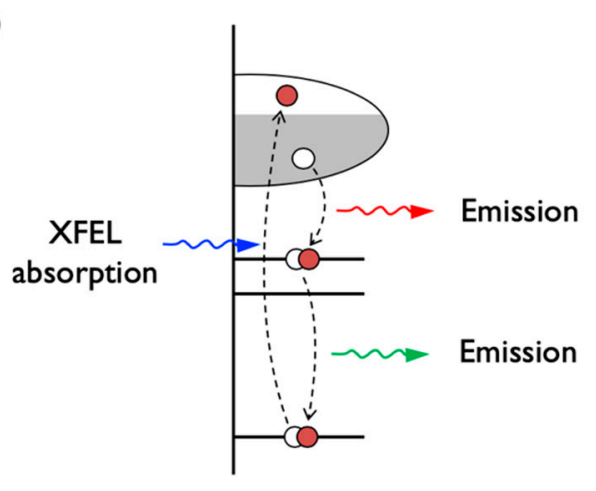

Figure 2. (a) General concept of experimental setup for the spectroscopic study of XFEL heated matter. An XFEL beam is tightly focused on a solid-density target to create warm/hot dense conditions. XES and XAS are the major diagnostics to be discussed in this paper. (b) Basic schemes of XES and XAS.

Considering typical XFEL parameters ( $1 \mathrm{keV}$ photon energy, $10^{12}$ photons in $30 \mathrm{fs}$ pulse, $1 \mu \mathrm{m}^{2}$ focal spot area), the corresponding electric field $\left(2 \times 10^{12} \mathrm{~V} / \mathrm{m}\right)$ is strong enough to induce nonlinear phenomena in atoms interacting with XFEL pulses. The photon number density in a cylinder volume corresponding to $1 \mu \mathrm{m}^{2}$ focal spot area and $9 \mu \mathrm{m}$ propagation length in $30 \mathrm{fs}$ is about $10^{29} \mathrm{~m}^{-3}$. It is similar to the typical atomic number densities of condensed matter (diamond: $1.76 \times 10^{29}$, water: $3.3 \times 10^{28} \mathrm{~m}^{-3}$ ), and the photon to atom ratio could be of the order of unity. Depending on X-ray photon energy and absorption, the energy density in excess of $1 \mathrm{eV} /$ atom as well as $10^{11} \mathrm{~J} / \mathrm{m}^{3}$ can be achieved.

To diagnose extreme light-matter interactions and different properties of the HED sample, various experimental techniques including imaging, diffractions, scattering, and spectroscopies with various wavelengths can be utilized. In this article, two spectroscopic techniques have been examined, namely, XES and XAS. The basic schemes of XES and XAS are illustrated in Figure $2 b$. These are complimentary 
to each other to probe occupied and unoccupied electronic states. With proper simulation on population kinetics in energy levels, various core-level processes and material properties of HED matter, such as resonant and non-resonant core-level transitions, collisions, continuum lowering, and opacity can be investigated [23-25].

\section{Intensity-Dependent EUV and X-ray Transmission}

In optical science, the intensity-dependent absorption or transmission of intense light are typical nonlinear responses of matter. Known as saturable absorption (SA) [26] and reverse saturable absorption (RSA) [27], these nonlinear phenomena are of great important owing to their wide range of applications in advanced laser and photonics technology. For example, SA preceded the invention of the laser; RSA and multi-photon absorption are applied in advanced microscopy, ultrafast optical switching, and laser pulse shaping [28,29].

In the short wavelength regimes, the advent of FEL has enabled the production of EUV and X-ray intensities high enough to deliver multiple photons to each atom in solid-density samples in tens of femtoseconds. Core-level transitions driven by a large number of X-ray photons can compete with and possibly modulated intrinsic atomic transitions in plasmas, such as Auger processes, fluorescence, and collisions, and nonlinear responses of matter can be expected.

In the course of exploring a new regime, aluminum is often considered an ideal element to begin with owing to its simple electronic structure. In ambient conditions, 10 electrons are bound $\left(1 s^{2} 2 s^{2} 2 p^{6}\right)$ and three are delocalized in the conduction band. The density of states (DOS) of these conduction electrons are close to those of free electron gas $\left(\sim E^{1 / 2}\right)$. The first experimental observation of the saturable absorption of EUV photons in aluminum foil was reported by Nagler et al. [30]. Photon energy $(92 \mathrm{eV})$ from the FLASH FEL is tuned above the L-edge $(73 \mathrm{eV})$ of cold $\mathrm{Al}$, and the main absorption channel is L-shell photoionization. After an L-electron is ejected from an atom, the L-edge of that particular ion increases to $93 \mathrm{eV}$, greater than photon energy. The lifetime of an L-hole via Auger decay is approximately $40 \mathrm{fs}$. Thus, the L-shell photoionization channel for singly ionized atoms will not be restored by the end of the pulse ( $15 \mathrm{fs})$. At high intensities, the fraction of such ions is high in the interaction volume, and photons in the later part of the pulse cannot be absorbed. These processes lead to an increase in EUV transmissions, as shown in Figure 3a. In other systems such as fused silica and tin, similar SA effects in the EUV regime have also been observed [31,32].
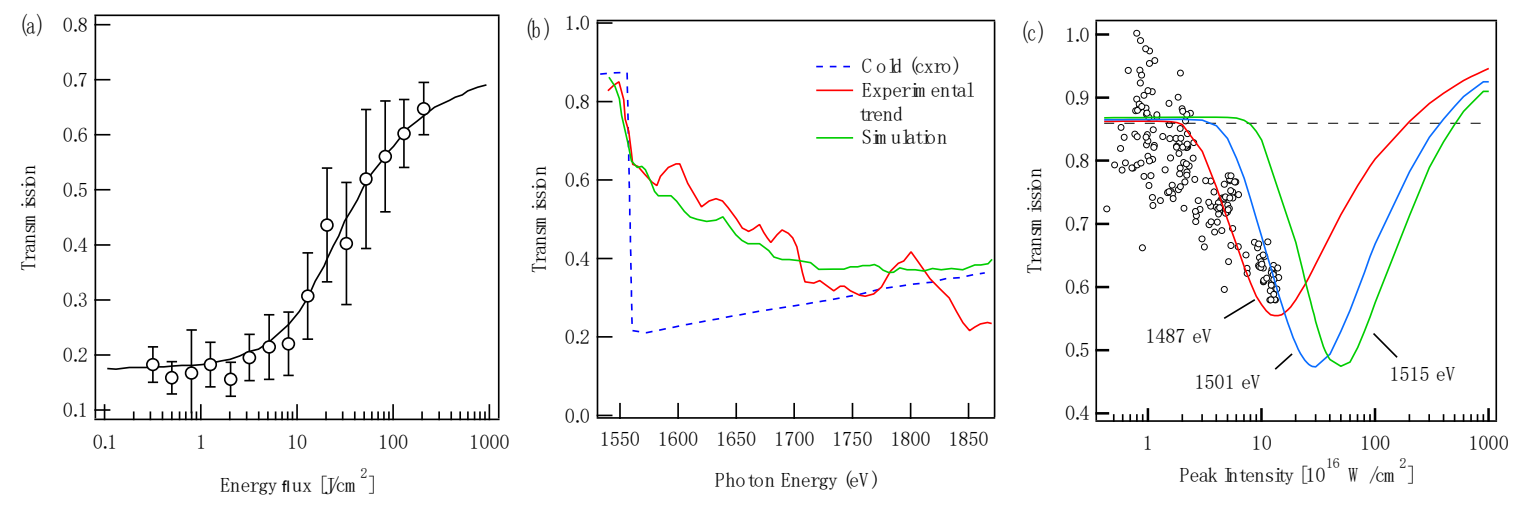

Figure 3. (a) Transmission of 53-nm-thick aluminum target as a function of $92 \mathrm{eV}$ photon fluence. The circles are the experimental data points, and the solid curve is the theoretical prediction [30]. (b) The transmission of 1- $\mu$ m-thick aluminum sample for the XFEL beam. The red line shows the experimental trend and the green line is from SCFLY simulations. The blue line indicates the cold transmission [33]. (c) The simulated transmission of 1.4- $\mu$ m-thick aluminum as a function of X-ray intensity with different photon energies (red: $1487 \mathrm{eV}$, blue $1501 \mathrm{eV}$, green: $1515 \mathrm{eV}$ ). Experimental measurements at $1487 \mathrm{eV}$ are also plotted via black circles [34]. Data in the figure are taken from [30,33,34] and replotted. 
The observation of the saturable absorption of X-ray photons can also be expected. Given that the core-hole lifetimes decrease with the increasing binding energies, the SA of the X-ray requires higher light intensities. In the soft X-ray regime, the SA of the LCLS pulse with a peak intensity of $10^{17} \mathrm{~W} / \mathrm{cm}^{2}$ was observed in aluminum [33]. As an XFEL pulse (1550 1850 eV) propagates through aluminum foil, the system is ionized to high charge states, and the absorption edge is shifted to higher energy. When the X-ray photon energy is tuned to above the K-edge of a specific ionization state, it is possible that in the middle of the pulse, no change state that can absorb a photon for K-shell photoionization occurs. As shown in Figure 3b, the increment of transmission is the most significant with photons just above the edge. High-energy photons can still be absorbed by higher charge states. In the hard X-ray regime, a similar effect was reported in iron [17]. The SACLA XFEL pulse $(>7113 \mathrm{eV})$ was focused to $50 \mathrm{~nm}$ to achieve a peak intensity of $1 \times 10^{20} \mathrm{~W} / \mathrm{cm}^{2}$ and the increased X-ray transmission beyond the light intensity $10^{18} \mathrm{~W} / \mathrm{cm}^{2}$. At this intensity, K-shell photoionization rate can compete with KLL Auger decay rate of mid-Z element.

As discussed in the case of SA, the excited states have smaller absorption than the ground states. In contrast, a nonlinear absorber in which the excited state absorption is larger than the ground state can undergo an opposite process, RSA. The first observation of decreasing X-ray transmission was also reported for an aluminum foil [35]. As shown in Figure 3c, tuned at K-L transition (1487 eV), which is below the K-edge of cold aluminum, XFEL transmission decreased in the intensity ranges of $10^{16}-10^{17} \mathrm{~W} / \mathrm{cm}^{2}$. Here, $\mathrm{X}$-ray photon energy is insufficient for a direct K-shell photoionization and the initial absorption channel of the ground state (K2L8) is L-shell photoionization. Although the ionization rate is small, a significant portion of atoms are excited to the K2L7 state owing to the large number of photons in the FEL pulse. The excited ion will absorb $1487 \mathrm{eV}$ photons effectively via resonant K-L excitation. Intense femtosecond XFEL pulse could deliver the second photon to the excited ion before it decays to the ground state in $40 \mathrm{fs}$, resulting in decreased transmission at higher intensity. With extensive collisional radiative population kinetic calculations, Cho, Chung, and Cho further discussed this phenomena in wide ranges of photon energy and intensity [34]. As shown in Figure 3c, at $1487 \mathrm{eV}$, a transition from RSA to SA is also predicted at higher intensities along with reproducing the experimental observations of RSA. RSA and the transition to SA can also occur at other resonant photon energies (1501 and $1515 \mathrm{eV}$ ) for higher charge states (K2L6 and K2L5). To produce multiple L-shell vacancies prior to the resonant excitation, higher XFEL intensities will be required.

In the SA experiment for iron, an improvement in the wave front of the transmitted hard X-ray FEL beam was observed [17]. Yoneda et al., also reported X-ray lasing at 1.5 Angstrom from $\mathrm{Cu}$ which was irradiated by intense two-color XFEL pulses [36]. These results give rise to the inference that similar to optical science, the nonlinear responses in short-wavelength regimes possess great potential for photonics applications.

\section{Isochoric Heating of Solid with X-ray}

Since the development of high-energy short-pulse lasers using the chirped pulse amplification (CPA) technique $[37,38]$, there have been many studies regarding the isochoric heating of solid density targets using femtosecond optical laser pulses [39-42]. However, these types of experiments have many difficulties that hamper the well-defined states of sample conditions. The optical laser deposits the energy in the skin depth (typically a few tens of nanometers) of material, and the heated sample with nanometer thickness releases very rapidly, often on the timescale of the laser pulse ( $100 \mathrm{fs})$. The interpretation of these experiments is further hindered because the time scale of the hydrodynamic expansion of the heated material is comparable with that of the electron-ion equilibration [43-45].

Therefore, it is highly desirable to explore the heating mechanisms of thicker layers of material using short bursts of energetic beams. If intense short pulses of X-ray at appropriate wavelengths are produced, a well-defined material with thickness in terms of micrometer can be heated to HED conditions in a controlled manner $[10,46,47]$. With the advent of XFELs, this concept has become 
feasible and provides attractive experimental platforms to study material properties and light-matter interactions in HED conditions.

The first experiment of its kind was conducted at the LCLS to heat aluminum with a focused XFEL pulse [16]. An aluminum foil with 1- $\mu \mathrm{m}$ thickness was irradiated with $80 \mathrm{fs}$ pulses at photon energies in the range of $1470-1830 \mathrm{eV}$ (below and above the Al K-edge). The peak intensity was $1.1 \times 10^{17} \mathrm{~W} / \mathrm{cm}^{2}$. Figure 4 shows the spectrally resolved $\mathrm{K} \alpha$ emission from various charge states as a function of the XFEL excitation photon energy.

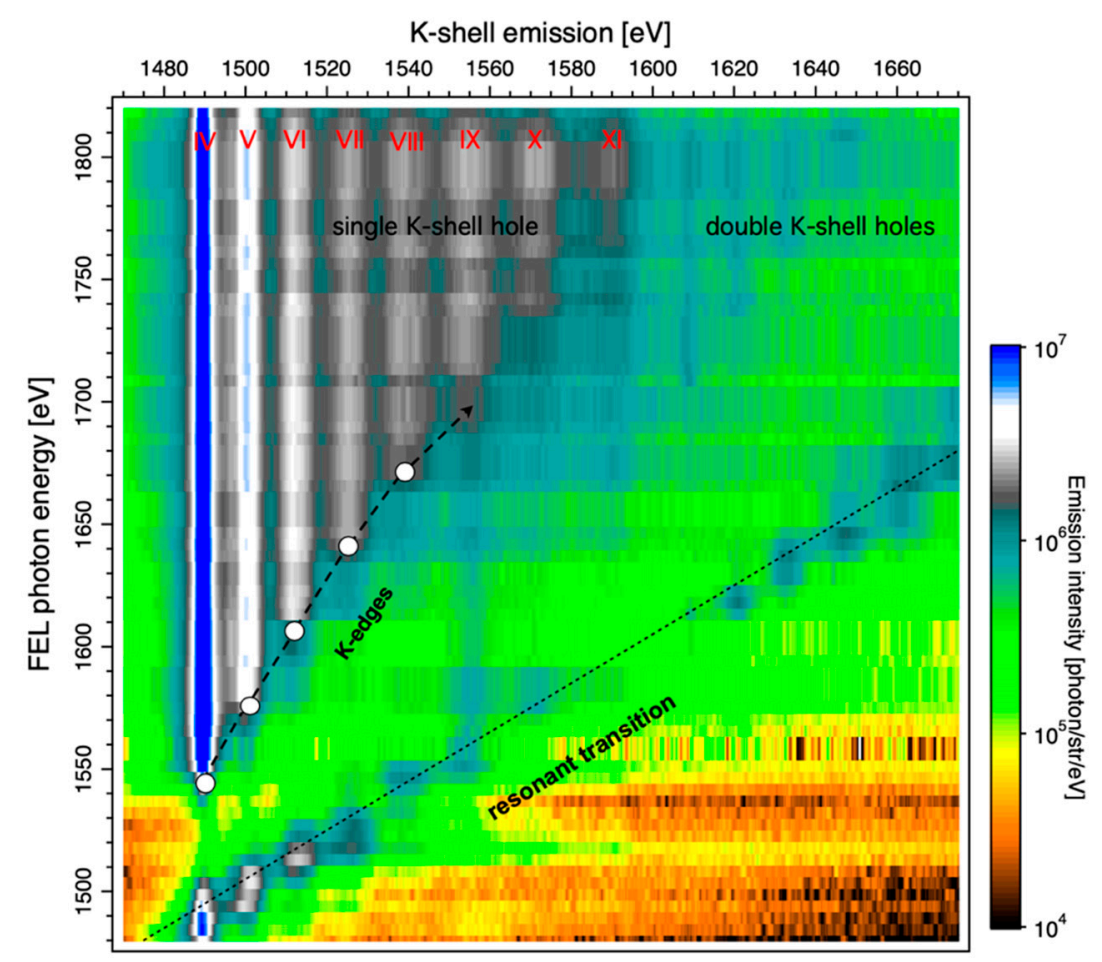

Figure 4. Spectrally resolved Al K $\alpha$ emission $(1460 \sim 1680 \mathrm{eV})$ as a function of XFEL excitation photon energy (1470 1825 eV). K-shell emission lines from Al charge states from 4+ to 11+ are marked. Emissions in the upper-left side of the graph are driven by the photoionization of a $\mathrm{K}$ electron to continuum by XFEL pulses. The onsets of emission lines correspondto the K-edge of charge states [16]. Bound-bound transitions can be observed as islands in the emission spectrum. Here, these correspond to the resonantly-driven $1 \mathrm{~s}-2 \mathrm{p}$ transitions [48]. Photoionization corresponds to driving the 1s electrons into some state in the continuum. Such transitions yield broadly flat emission intensities over an extended range of FEL wavelengths, observed experimentally at higher FEL photon energies. The data in the figure are taken from the $[16,48]$ and replotted.

When the XFEL photon energy is above the K-edge $(1560 \mathrm{eV})$, the initially dominant process is $\mathrm{K}$-shell photoionization. Once created, a K-shell vacancy decays either radiatively or non-radiatively. In the radiative process, a core-hole is mainly filled by an L-electron ( $2 \mathrm{p}-1 \mathrm{~s}$ transition) and a K $\alpha$ photon is emitted. Though this fluorescent rate is small $(4 \%)$ in $\mathrm{Al}$, owing to the large number of incident photons in an FEL pulse, a single-shot XES measurement is feasible. K $\alpha$ emission lines from charge states $4+$ to $11+$ are observed, indicating the formation of highly-ionized plasmas. The dominant $(96 \%)$ recombination process of a core-hole is non-radiative KLL Auger decay. A K-hole is filled with an L-electron while another L-electron is emitted, and the level of ionization is increased. Energetic Auger electrons have large collisional rates and quickly thermalize with free electrons.

The collision of free electrons at a high temperature dominates the ioniation dynamics and determines the charge state distribution in the system [49]. The processes happen in a duration of pulse to ensure that the electronic system is effectively heated by an XFEL. However, the electron-ion relaxation and hydrodynamic expansion of the sample do not take place yet, and an isochoric condition 
can be achieved. Using the collisional-radiative population kinetic calculations, which were consistent with the observed spectral emissions, the creation of solid density $\mathrm{Al}$ with an electron temperature over $100 \mathrm{eV}$ and electron densities of $5 \times 10^{23} \mathrm{~cm}^{-3}$ ( 3× of the ambient Al) was reported [16].

In contrast to the above edge pumping of K-electons, the XFEL photons with energies below the $\mathrm{K}$ abosrption edge can be absorbed only by L-shell and free electrons, and no K-shell vacancy was expected. However, a rich array of $\mathrm{K} \alpha$ emissions (marked as resonant transitions in Figure 4) was also observed by tuning the LCLS pulse to photon energies below the K absorption edge [48]. This unexpected observation can be achieved via sequential multi-photon absorption and the inner-shell resonant processes driven by an intense X-ray pulse. Even though the cross-section is small, multiple $\mathrm{X}$-ray photons in a single pulse are sequentially absorbed in the same atom and ionize L-electrons. Then, a photon resonantly excites a K-electron into an L-hole (1s-2p transition) and opens up a channel for $K \alpha$ production. Charge states resonating with $X$-ray photons are both single $\left(1 s^{1} 2 p^{n}\right)$ and double ( $1 \mathrm{~s}^{0} 2 \mathrm{p}^{\mathrm{n}}$, hallow ions) K-hole states, indicating exotic plasma conditions. Owing to this new $\mathrm{X}$-ray-matter interaction channel, the sample can be heated to more than $50 \mathrm{eV}$ with pumping below the K absorption edge [48].

Isochoric heating with intense FEL has also been applied to other elements. Zastrau et al. reported the ultrafast heating of cryogenic hydrogen using FLASH EUV FEL pulses and studied the structural transition from dense cryogenic molecules to a nearly uncorrelated plasma-like structure [22]. Medvedev and Ziaja investigated the multi-step structural transitions of diamond (diamond $\rightarrow$ graphite-like $\rightarrow$ WDM) irradiated by SACLA XFEL pulses [13]. Levy et al. used an LCLS XFEL pulse to heat a silver foil and probed the hydrodynamic expansion of the front and rear surfaces of the sample to investigate the uniformity of energy deposition [50]. Williams et al., also used an LCLS pulse to heat aluminum foil and studied the transient optical properties in the XUV regime [51]. These isochoric heating experiments were compared with various simulations and models, showing that valuable benchmark systems to study different properties of matter under extreme conditions can be created.

\section{Continuum Lowering in HED Plasmas}

The continuum lowering, also known as ionization potential depression (IPD), is a fundamental component of dense plasma studies. The electric fields of plasma compete with the atomic field, particularly for the higher-lying levels. The orbital electrons may not be bound for certain states and the effective ionization energies are reduced. It affects the ionization balance, excited state populations, electron density, as well as temperature. The opacity of emission and the absorption lines are also affected. Despite its importance, the formulation of IPD was based on crude methods. In the widely accepted classical model by Stewart and Pyatt (SP) [52], the radii for the lower bound of the continuum are estimated for two extreme cases, very dilute and dense plasmas, using the Debye radius and ion sphere radius, respectively. Then, IPD for different conditions was formulated via bridging the two cases. However, this model has been adopted without rigorous tests at various conditions owing to many obstacles in accurate measurement during the experiment.

Given that the Al K-shell emission shown in Figure 4 originates from the absorption of LCLS XFEL photons by specific ions, which have unoccupied states and allow the transitions of electrons from the K-shell, XES in this measurement can also be considered as XAS in the fluorescence yield mode. This technique is sensitive to determine the lowest energy transition from core-level to unoccupied DOS, i.e., the absorption edge. In Figure 4, as the FEL photon energy progressively increases, the onsets of emission lines from IV to higher charge states are observed. Ciricosta et al. identified these emission thresholds as the K-edges of charge states IV VII, given by the energy difference between the K-shell binding energies and IPDs [53,54].

As shown in Figure 5, in plasma conditions of solid-density and about $100 \mathrm{eV}$ temperature, these results provided the first experimental benchmarks to test the SP model, and significant mismatches were found. Interestingly, a modified but less attentive version of continuum lowering 
model by Ecker and Kröll [EK] [55] provided better consistency. This test has stirred up old interests in the topic. At the Orion laser facility, IPDs in a-few-times-compressed aluminum plasmas with $500 \sim 700 \mathrm{eV}$ were also measured. Here, the measured K-shell emissions were reasonably consistent with the spectrum calculation using the SP model but they were significantly more consistent with the simple ion sphere model, while the EK model was not satisfactory [56,57]. These conflicting results have led to the resurgence of the theoretical work for IPD with modern tools for modeling. For example, Son et al. developed a two-step Hartree-Fock-Slater model, which is claimed to yield a better consistency with both the Orion and LCLS results [58]. Vinko et al., performed calculations using a finite-temperature density functional theory (DFT) for the electronic structure of dense aluminum, which shows good consistency with the LCLS experiment but not with the Orion experiment [59]. As shown in Figure 5, while several theoretical predictions by various groups are diverging, it is apparent that more benchmark data for various HED plasma conditions are required, and the FELs and X-ray spectroscopy techniques would be critical tools to guide future investigations.

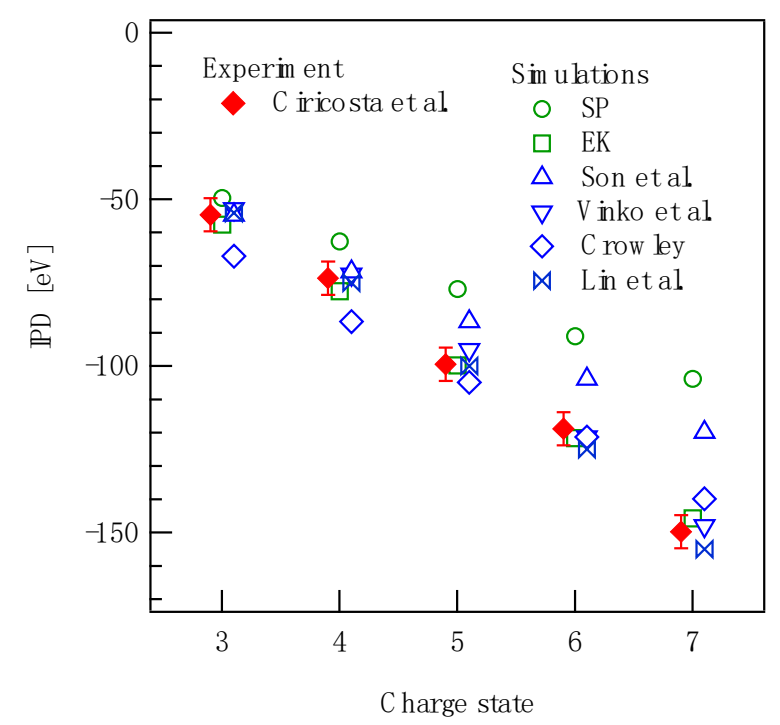

Figure 5. Comparison of IPD in solid-density aluminum plasmas. The classical models of SP [52] and EK [55] (green markers), and the modern calculations by Son et al. [58], Vinko et al. [59], Crowley [60], and Lin et al. [61] (blue markers) are compared with the experimental results by Ciricosta et al. [53] (red markers). A reasonable agreement is found only for the ground state (Al 3+). Diverging results for higher charge states illustrate the difficulties in theoretical work even with modern computational tools. The data in the figure are taken from the $[53,58-61]$ and replotted.

\section{Conclusions}

Core-level transitions driven by the tightly focused X-ray and EUV FELs can compete with and possibly modulate intrinsic atomic transitions in plasmas, such as Auger processes, fluorescence, and collisions, thereby driving the nonlinear responses of matter. SA i.e., increased transmission at higher intensity, has been observed in a broad range of energies and materials such as EUV, soft X-ray in aluminum [30,33], and hard X-ray in iron [17]. Irrespective of the photon energies and elements, photoionization by FELs at the above absorption edges are fast enough to deplete the ground states. An opposite trend of response (RSA) for soft X-ray was also reported in aluminum [35]. At the resonant core-level transition below the absorption edge, decreased $\mathrm{X}$-ray transmission was observed in aluminum. These nonlinear responses in short-wavelength regime are of great importance in photonic applications such as improving the wave front of FEL beam [17] and the development of atomic X-ray lasers [36].

The isochoric heating of a solid target with an intense femtosecond FEL pulse is proved to be an experimental platform to create a large volume of high-energy-density matter with well-defined energy 
densities in a wide range of parameters, which include hot-dense (e.g., aluminum at a temperature of $100 \mathrm{eV}$ [16]) and warm-dense conditions (e.g., carbon at 6-10 eV [13] and silver at 10-15 eV [50] at their initial solid densities). In comparison to nanometer-thick targets heated with an optical laser for reasonable uniformity, the use of X-ray FEL allows to heat micron-thick targets with improved uniformity. It provides great advantage in the investigation of a bulk of HED matter in longer confinement periods. The measurement of $\mathrm{X}$-ray emission and absorption spectra are complementary to probe both the occupied and unoccupied electronic states, which affect basic plasma parameters such as ionization potential and temperature, as well as the transport properties such as optical and thermal conductivities in HED conditions.

The measurement of K-shell emission spectra for the XFEL-heated matter has yielded the first experimental measurement of IPD in aluminum with 100 200 eV temperature and solid density. The precise control of XFEL photon energy allows to determine the K-edges and the continuum lowering of various $\mathrm{Al}$ charge states in such plasma conditions within a few $\mathrm{eV}$. The FEL experiments provide accurate benchmark data for re-evaluating existing IPD models and guiding the further theoretical and computational advances on this fundamental but challenging subject of HED matter.

With further development of FEL technology, X-ray pulses with attosecond duration, narrow bandwidth, and temporal coherence are also available and open new opportunities to study material properties and light-matter interactions in extreme conditions. For example, nonlinear X-ray spectroscopic technique, such as resonant inelastic X-ray scattering (RIXS) using attosecond coherent XFEL pulses shed light on the direct observation of relocalization of electronic wave packets excited from core-levels in the HED plasma environment. New sets of experimental data in femtosecond and nanometer regimes with advanced computation and theories will afford to not only address long standing questions, but also find new outstanding ones in plasma physics, fusion science, as well as X-ray optics.

Funding: The author was supported by the Institute for Basic Science (IBS-R012-D1) and the National Research Foundation (NRF2015R1A5A1009962 and NRF-2019R1A2C2002864) of Korea.

Conflicts of Interest: The author declares no conflict of interest. The funders had no role in the design of the study; in the collection, analyses, or interpretation of data; in the writing of the manuscript, or in the decision to publish the results.

\section{References}

1. Ackermann, W.; Asova, G.; Ayvazyan, V.; Azima, A.; Baboi, N.; Bähr, J.; Balandin, V.; Beutner, B.; Brandt, A.; Bolzmann, A.; et al. Operation of a free-electron laser from the extreme ultraviolet to the water window. Nat. Photonics 2007, 1, 336. [CrossRef]

2. Emma, P.; Akre, R.; Arthur, J.; Bionta, R.; Bostedt, C.; Bozek, J.; Brachmann, A.; Bucksbaum, P.; Coffee, R.; Decker, F.J.; et al. First lasing and operation of an angstrom-wavelength free-electron laser. Nat. Photonics 2010, 4, 641-647. [CrossRef]

3. Ishikawa, T.; Aoyagi, H.; Asaka, T.; Asano, Y.; Azumi, N.; Bizen, T.; Ego, H.; Fukami, K.; Fukui, T.; Furukawa, Y.; et al. A compact X-ray free-electron laser emitting in the sub-angstrom region. Nat. Photonics 2012, 6, 540-544. [CrossRef]

4. Kang, H.-S.; Min, C.-K.; Heo, H.; Kim, C.; Yang, H.; Kim, G.; Nam, I.; Baek, S.Y.; Choi, H.-J.; Mun, G.; et al. Hard X-ray free-electron laser with femtosecond-scale timing jitter. Nat. Photonics 2017, 11, 708-713. [CrossRef]

5. Daligault, J.; Gupta, S. Electron-Ion Scattering in Dense Multi-Component Plasmas: Application to the Outer Crust of an Accreting Neutron Star. Astrophys. J. 2009, 703, 994-1011. [CrossRef]

6. Glenzer, S.H.; MacGowan, B.J.; Michel, P.; Meezan, N.B.; Suter, L.J.; Dixit, S.N.; Kline, J.L.; Kyrala, G.A.; Bradley, D.K.; Callahan, D.A.; et al. Symmetric inertial confinement fusion implosions at ultra-high laser energies. Science 2010, 327, 1228-1231. [CrossRef] [PubMed]

7. Chan, J.W.; Huser, T.; Risbud, S.; Krol, D.M. Structural changes in fused silica after exposure to focused femtosecond laser pulses. Opt. Lett. 2001, 26, 1726-1728. [CrossRef] [PubMed] 
8. National Research Council. Frontiers in High Energy Density Physics: The X-Games of Contemporary Science; The National Academies Press: Washington, DC, USA, 2003; pp. 1-176.

9. U.S. Department of Energy, Office of Science and National Nuclear Security Administration. Basic Research Needs for High Energy Density Laboratory Physics; U.S. Department of Energy: Washington, DC, USA, 2010.

10. Lee, R.W.; Moon, S.J.; Chung, H.-K.; Rozmus, W.; Baldis, H.A.; Gregori, G.; Cauble, R.C.; Landen, O.L.; Wark, J.S.; $\mathrm{Ng}$, A.; et al. Finite temperature dense matter studies on next-generation light sources. J. Opt. Soc. Am. B 2003, 20, 770-778. [CrossRef]

11. Lee, R.W.; Baldis, H.A.; Cauble, R.C.; Landen, O.L.; Wark, J.S.; Ng, A.; Rose, S.J.; Lewis, C.; Riley, D.; Gauthier, J.C.; et al. Plasma-based studies with intense X-ray and particle beam sources. Laser Part. Beams 2002, 20, 527-536. [CrossRef]

12. Beckwith, M.A.; Jiang, S.; Schropp, A.; Fernandez-Pañella, A.; Rinderknecht, H.G.; Wilks, S.C.; Fournier, K.B.; Galtier, E.C.; Xing, Z.; Granados, E.; et al. Imaging at an x-ray absorption edge using free electron laser pulses for interface dynamics in high energy density systems. Rev. Sci. Instrum. 2017, 88, 053501. [CrossRef]

13. Medvedev, N.; Ziaja, B. Multistep transition of diamond to warm dense matter state revealed by femtosecond X-ray diffraction. Sci. Rep. 2018, 8, 5284. [CrossRef] [PubMed]

14. Brown, C.R.D.; Gericke, D.O.; Cammarata, M.; Cho, B.I.; Döppner, T.; Engelhorn, K.; Förster, E.; Fortmann, C.; Fritz, D.; Galtier, E.; et al. Evidence for a glassy state in strongly driven carbon. Sci. Rep. 2014, 4, 5214. [CrossRef] [PubMed]

15. Fletcher, L.B.; Lee, H.J.; Doppner, T.; Galtier, E.; Nagler, B.; Heimann, P.; Fortmann, C.; LePape, S.; Ma, T.; Millot, M.; et al. Ultrabright X-ray laser scattering for dynamic warm dense matter physics. Nat. Photonics 2015, 9, 274-279. [CrossRef]

16. Vinko, S.M.; Ciricosta, O.; Cho, B.I.; Engelhorn, K.; Chung, H.K.; Brown, C.R.; Burian, T.; Chalupsky, J.; Falcone, R.W.; Graves, C.; et al. Creation and diagnosis of a solid-density plasma with an X-ray free-electron laser. Nature 2012, 482, 59-62. [CrossRef]

17. Yoneda, H.; Inubushi, Y.; Yabashi, M.; Katayama, T.; Ishikawa, T.; Ohashi, H.; Yumoto, H.; Yamauchi, K.; Mimura, H.; Kitamura, H. Saturable absorption of intense hard X-rays in iron. Nat. Commun. 2014, 5, 5080. [CrossRef]

18. Mukamel, S.; Healion, D.; Zhang, Y.; Biggs, J.D. Multidimensional Attosecond Resonant X-Ray Spectroscopy of Molecules: Lessons from the Optical Regime. Ann. Rev. Phys. Chem. 2013, 64, 101-127. [CrossRef]

19. Bennett, K.; Zhang, Y.; Kowalewski, M.; Hua, W.; Mukamel, S. Multidimensional resonant nonlinear spectroscopy with coherent broadband x-ray pulses. Phys. Scr. 2016, T169, 014002. [CrossRef]

20. Mimura, H.; Yumoto, H.; Matsuyama, S.; Koyama, T.; Tono, K.; Inubushi, Y.; Togashi, T.; Sato, T.; Kim, J.; Fukui, R.; et al. Generation of $1020 \mathrm{~W} \mathrm{~cm}-2$ hard X-ray laser pulses with two-stage reflective focusing system. Nat. Commun. 2014, 5, 3539. [CrossRef]

21. Ping, Y.; Fernandez-Panella, A.; Sio, H.; Correa, A.; Shepherd, R.; Landen, O.; London, R.A.; Sterne, P.A.; Whitley, H.D.; Fratanduono, D.; et al. Differential heating: A versatile method for thermal conductivity measurements in high-energy-density matter. Phys. Plasmas 2015, 22, 092701. [CrossRef]

22. Zastrau, U.; Sperling, P.; Harmand, M.; Becker, A.; Bornath, T.; Bredow, R.; Dziarzhytski, S.; Fennel, T.; Fletcher, L.B.; Förster, E.; et al. Resolving Ultrafast Heating of Dense Cryogenic Hydrogen. Phys. Rev. Lett. 2014, 112, 105002. [CrossRef]

23. Chung, H.K.; Chen, M.H.; Morgan, W.L.; Ralchenko, Y.; Lee, R.W. Flychk: Generalized population kinetics and spectral model for rapid spectroscopic analysis for all elements. High Energy Density Phys. 2005, 1, 3-12. [CrossRef]

24. Chung, H.K.; Chen, M.H.; Lee, R.W. Extension of atomic configuration sets of the Non-LTE model in the application to the $\mathrm{K} \alpha$ diagnostics of hot dense matter. High Energy Density Phys. 2007, 3, 57-64. [CrossRef]

25. Chung, H.K.; Cho, B.I.; Ciricosta, O.; Vinko, S.M.; Wark, J.S.; Lee, R.W. Atomic processes modeling of X-ray free electron laser produced plasmas using SCFLY code. AIP Conf. Proc. 2017, 1811, 020001.

26. Lewis, G.N.; Lipkin, D.; Magel, T.T. Reversible Photochemical Processes in Rigid Media. A Study of the Phosphorescent State. J. Am. Chem. Soc. 1941, 63, 3005-3018. [CrossRef]

27. Wood, G.L.; Clark, W.W., III; Miller, M.J.; Salamo, G.J.; Sharp, E.J. Evaluation Of Passive Optical Limiters And Switches; SPIE 1989 Technical Symposium on Aerospace Sensing; SPIE: Bellingham, WA, USA, 1989; Volume 1105. 
28. Zhang, B.; Li, Y.; Liu, R.; Pritchett, T.M.; Haley, J.E.; Sun, W. Extending the bandwidth of reverse saturable absorption in platinum complexes using two-photon-initiated excited-state absorption. ACS Appl. Mater. Interfaces 2013, 5, 565-572. [CrossRef]

29. He, G.S.; Tan, L.S.; Zheng, Q.; Prasad, P.N. Multiphoton absorbing materials: Molecular designs, characterizations, and applications. Chem. Rev. 2008, 108, 1245-1330. [CrossRef]

30. Nagler, B.; Zastrau, U.; Faustlin, R.R.; Vinko, S.M.; Whitcher, T.; Nelson, A.J.; Sobierajski, R.; Krzywinski, J.; Chalupsky, J.; Abreu, E.; et al. Turning solid aluminium transparent by intense soft $\mathrm{X}$-ray photoionization. Nat. Phys. 2009, 5, 693-696.

31. Inubushi, Y.; Yoneda, H.; Higashiya, A.; Ishikawa, T.; Kimura, H.; Kumagai, T.; Morimoto, S.; Nagasono, M.; Ohashi, H.; Sato, F.; et al. Measurement of saturable absorption by intense vacuum ultraviolet free electron laser using fluorescent material. Rev. Sci. Instrum. 2010, 81, 036101. [CrossRef]

32. Yoneda, H.; Inubushi, Y.; Sato, F.; Morimoto, S.; Kumagaya, T.; Nagasono, M.; Higashiya, A.; Yabashi, M.; Ishikawa, T.; Ohashi, H.; et al. Observation of Saturable Absorption of Sn Metal Film with Intense EUV Laser Pulse; Ultrafast Nonlinear Optics; SPIE: Bellingham, WA, USA, 2009; Volume 7501.

33. Rackstraw, D.S.; Ciricosta, O.; Vinko, S.M.; Barbrel, B.; Burian, T.; Chalupsky, J.; Cho, B.I.; Chung, H.K.; Dakovski, G.L.; Engelhorn, K.; et al. Saturable absorption of an x-ray free-electron-laser heated solid-density aluminum plasma. Phys. Rev. Lett. 2015, 114, 015003. [CrossRef]

34. Cho, M.S.; Chung, H.K.; Cho, B.I. Intensity-dependent resonant transmission of x-rays in solid-density aluminum plasma. Phys. Plasmas 2018, 25, 053301. [CrossRef]

35. Cho, B.I.; Cho, M.S.; Kim, M.; Chung, H.K.; Barbrel, B.; Engelhorn, K.; Burian, T.; Chalupsky, J.; Ciricosta, O.; Dakovski, G.L.; et al. Observation of Reverse Saturable Absorption of an X-ray Laser. Phys. Rev. Lett. 2017, 119, 075002. [CrossRef] [PubMed]

36. Yoneda, H.; Inubushi, Y.; Nagamine, K.; Michine, Y.; Ohashi, H.; Yumoto, H.; Yamauchi, K.; Mimura, H.; Kitamura, H.; Katayama, T.; et al. Atomic inner-shell laser at 1.5-ångström wavelength pumped by an X-ray free-electron laser. Nature 2015, 524, 446. [CrossRef] [PubMed]

37. Strickland, D.; Mourou, G. Compression of amplified chirped optical pulses. Opt. Commun. 1985, 56, $219-221$. [CrossRef]

38. Mourou, G. The ultrahigh-peak-power laser: Present and future. Appl. Phys. B 1997, 65, 205-211. [CrossRef]

39. Forsman, A.; Ng, A.; Chiu, G.; More, R.M. Interaction of femtosecond laser pulses with ultrathin foils. Phys. Rev. E 1998, 58, R1248-R1251. [CrossRef]

40. Audebert, P.; Shepherd, R.; Fournier, K.B.; Peyrusse, O.; Price, D.; Lee, R.; Springer, P.; Gauthier, J.C.; Klein, L. Heating of Thin Foils with a Relativistic-Intensity Short-Pulse Laser. Phys. Rev. Lett. 2002, 89, 265001. [CrossRef]

41. Ng, A.; Ao, T.; Perrot, F.; Dharma-Wardana, M.W.C.; Foord, M.E. Idealized slab plasma approach for the study of warm dense matter. Laser Part. Beams 2005, 23, 527-537. [CrossRef]

42. Ao, T.; Ping, Y.; Widmann, K.; Price, D.F.; Lee, E.; Tam, H.; Springer, P.T.; Ng, A. Optical properties in nonequilibrium phase transitions. Phys. Rev. Lett. 2006, 96, 055001. [CrossRef]

43. Spitzer, L., Jr. Physics of Fully Ionized Gases; Dover Publications: Wiley, NY, USA, 1956.

44. Cho, B.I.; Engelhorn, K.; Correa, A.A.; Ogitsu, T.; Weber, C.P.; Lee, H.J.; Feng, J.; Ni, P.A.; Ping, Y.; Nelson, A.J.; et al. Electronic structure of warm dense copper studied by ultrafast x-ray absorption spectroscopy. Phys. Rev. Lett. 2011, 106, 167601. [CrossRef]

45. Cho, B.I.; Ogitsu, T.; Engelhorn, K.; Correa, A.A.; Ping, Y.; Lee, J.W.; Bae, L.J.; Prendergast, D.; Falcone, R.W.; Heimann, P.A. Measurement of Electron.-Ion. Relaxation in Warm Dense Copper. Sci. Rep. 2016, 6, 18843. [CrossRef]

46. Dyer, G.; Sheppherd, R.; Kuba, J.; Fill, E.; Wootton, A.; Patel, P.; Price, D.; Ditmire, T. Isochoric heating of solid aluminium with picosecond X-ray pulses. J. Mod. Opt. 2003, 50, 2495-2505. [CrossRef]

47. Tschentscher, T.; Toleikis, S. Investigations of ultrafast phenomena in high-energy density physicsusing X-ray FEL radiation. Eur. Phys. J. D At. Mol. Opt. Plasma Phys. 2005, 36, 193-197.

48. Cho, B.I.; Engelhorn, K.; Vinko, S.M.; Chung, H.K.; Ciricosta, O.; Rackstraw, D.S.; Falcone, R.W.; Brown, C.R.; Burian, T.; Chalupsky, J.; et al. Resonant Kalpha spectroscopy of solid-density aluminum plasmas. Phys. Rev. Lett. 2012, 109, 245003. [CrossRef] [PubMed] 
49. Vinko, S.M.; Ciricosta, O.; Preston, T.R.; Rackstraw, D.S.; Brown, C.R.D.; Burian, T.; Chalupský, J.; Cho, B.I.; Chung, H.K.; Engelhorn, K.; et al. Investigation of femtosecond collisional ionization rates in a solid-density aluminium plasma. Nat. Commun. 2015, 6, 6397. [CrossRef]

50. Lévy, A.; Audebert, P.; Shepherd, R.; Dunn, J.; Cammarata, M.; Ciricosta, O.; Deneuville, F.; Dorchies, F.; Fajardo, M.; Fourment, C.; et al. The creation of large-volume, gradient-free warm dense matter with an x-ray free-electron laser. Phys. Plasmas 2015, 22, 030703. [CrossRef]

51. Williams, G.O.; Künzel, S.; Daboussi, S.; Iwan, B.; Gonzalez, A.I.; Boutu, W.; Hilbert, V.; Zastrau, U.; Lee, H.J.; Nagler, B.; et al. Tracking the ultrafast XUV optical properties of $x$-ray free-electron-laser heated matter with high-order harmonics. Phys. Rev. A 2018, 97, 023414. [CrossRef]

52. Stewart, J.C.; Pyatt, K.D., Jr. Lowering of Ionization Potentials in Plasmas. Astrophys. J. 1966, $144,1203$. [CrossRef]

53. Ciricosta, O.; Vinko, S.M.; Chung, H.K.; Cho, B.I.; Brown, C.R.; Burian, T.; Chalupsky, J.; Engelhorn, K.; Falcone, R.W.; Graves, C.; et al. Direct measurements of the ionization potential depression in a dense plasma. Phys. Rev. Lett. 2012, 109, 065002. [CrossRef]

54. Ciricosta, O.; Vinko, S.M.; Chung, H.K.; Jackson, C.; Lee, R.W.; Preston, T.R.; Rackstraw, D.S.; Wark, J.S. Detailed model for hot-dense aluminum plasmas generated by an x-ray free electron laser. Phys. Plasmas 2016, 23, 022707. [CrossRef]

55. Ecker, G.; Kröll, W. Lowering of the Ionization Energy for a Plasma in Thermodynamic Equilibrium. Phys. Fluids 1963, 6, 62-69. [CrossRef]

56. Hoarty, D.J.; Allan, P.; James, S.F.; Brown, C.R.D.; Hobbs, L.M.R.; Hill, M.P.; Harris, J.W.O.; Morton, J.; Brookes, M.G.; Shepherd, R.; et al. The first data from the Orion laser; measurements of the spectrum of hot, dense aluminium. High Energy Density Phys. 2013, 9, 661-671. [CrossRef]

57. Hoarty, D.J.; Allan, P.; James, S.F.; Brown, C.R.D.; Hobbs, L.M.R.; Hill, M.P.; Harris, J.W.O.; Morton, J.; Brookes, M.G.; Shepherd, R.; et al. Observations of the Effect of Ionization-Potential Depression in Hot Dense Plasma. Phys. Rev. Lett. 2013, 110, 265003. [CrossRef] [PubMed]

58. Son, S.-K.; Thiele, R.; Jurek, Z.; Ziaja, B.; Santra, R. Quantum-Mechanical Calculation of Ionization-Potential Lowering in Dense Plasmas. Phys. Rev. X 2014, 4, 031004. [CrossRef]

59. Vinko, S.M.; Ciricosta, O.; Wark, J.S. Density functional theory calculations of continuum lowering in strongly coupled plasmas. Nat. Commun. 2014, 5, 3533. [CrossRef] [PubMed]

60. Crowley, B.J.B. Continuum lowering-A new perspective. High Energy Density Phys. 2014, 13, 84-102. [CrossRef]

61. Lin, C.; Röpke, G.; Kraeft, W.-D.; Reinholz, H. Ionization-potential depression and dynamical structure factor in dense plasmas. Phys. Rev. E 2017, 96, 013202. [CrossRef] [PubMed] 